\title{
Numerical Study of Wall Pressure Fluctuations for Zero and Non-Zero Pressure Gradient Turbulent Boundary Layers
}

\author{
Nan $\mathrm{Hu}^{*}$, Christina Appel ${ }^{\dagger}$, Michaela Herr ${ }^{\ddagger}$, Nils Reiche ${ }^{\S}$ and Roland Ewert
}

DLR, Technical Acoustics Branch, D-38108 Braunschweig, Germany

\begin{abstract}
Turbulent boundary layers on a flat plate configuration are simulated using synthetic turbulence generated by the Fast Random Particle-Mesh Method. The averaged turbulence statistics needed for the stochastic realization is provided by a Reynolds averaged Navier-Stokes calculation. Wall pressure fluctuations are obtained by calculating a Poisson equation including both the mean-shear turbulence interaction source term and the turbulence-turbulence interaction source term. The Poisson equation is solved by means of Hockney's method. Wall pressure fluctuations for zero and adverse pressure gradient boundary layers are calculated. The adverse pressure gradient is realized by placing an airfoil above the flat plate. Simulated one-point spectra and two-point statistics are analyzed. The results are compared to the experimental results, which were measured in the Acoustic Windtunnel Braunschweig for the same configurations. Good agreement with the experimental results is obtained.
\end{abstract}

\section{Introduction}

Investigation of wall pressure fluctuations beneath a turbulent boundary layer is a fundamental topic. The major concern is the flow-induced vibration and the resulting sound generation, which for example play an essential role for aircraft cabin noise. In general, not only the excitation power but also the spatial and temporal properties of the wall pressure fluctuations are relevant for the resulting vibration. The correlation decay in longitudinal and lateral direction and the convective velocity of the surface fluctuating pressure pattern are the most relevant features for representing its spatial and temporal properties. A comprehensive overview on the subject of wall pressure fluctuations, the structural response and the induced sound radiation was given in the monograph of Blake. ${ }^{1}$ Many empirical spectral models were proposed. One of the most frequently cited model is from the work of Goody, ${ }^{2}$ which was derived from experimental results for zero pressure gradient (ZPG) turbulent boundary layers. To represent the spatial and temporal properties of the fluctuating pressure field, $\operatorname{Corcos}^{3}$ proposed exponential functions by means of empirical coherence decay parameters of both longitudinal and lateral directions and the phase velocities. Several fundamental experiments have been carried out to measure the characteristics of the wall pressure fluctuations, e.g. refer to the work of Willmarth \& Wooldridge, Bull, Blake and Farabee \& Casarella. ${ }^{4-7}$ However, a precise and extensive measurement of the fluctuating pressure field relevant for structural excitation is difficult, e.g. due to a finite sensor size and the limitation of the experimental facilities. In more recent studies, Arguillat et al. ${ }^{8}$ and Ehrenfried \& Koop ${ }^{9}$ measured the wavenumberfrequency spectra by using array technology. Although in their measurements the convective ridge and the acoustic part were well identified, the obtained results e.g. the spectra in the low wavenumber domain were still not conclusive due to the experimental difficulties indicated above.

Due to the development of supercomputers, a numerical study of the fluctuating pressure field has become possible. Work of direct numerical simulation and large eddy simulation to this topic has been

\footnotetext{
*Research Engineer, AIAA member, nan.hu@dlr.de

${ }^{\dagger}$ Research Engineer, AIAA member, christina.appel@dlr.de

${ }^{\ddagger}$ Research Engineer, AIAA senior member, michaela.herr@dlr.de

$\S$ Research Engineer, AIAA member, nils.reiche@dlr.de

`Research Engineer, AIAA senior member, roland.ewert@dlr.de
} 
published by many authors. ${ }^{10-14}$ However, due to the extremely expensive computational resources, applications are generally restricted to generic studies at low Reynolds number.

Hu et al. ${ }^{15}$ investigated a high Reynolds number generic boundary layer with an effective numerical procedure. The fluctuating pressure was calculated by solving a Poisson equation. The synthetic source terms on the right-hand side of the Poisson equation was generated by the Fast Random Particle-Mesh Method (FRPM). Both mean-shear turbulence and turbulence-turbulence terms were considered. The equation was solved by using a free-space Green function and solving the convolution with a spatial fast Fourier transform utilizing Hockney's method. ${ }^{16}$ The features of the wall pressure fluctuations including the wavenumber-frequency spectra were analyzed in detail. Good agreement with the existing results from literature was achieved.

In the present work, ZPG boundary layers with two different velocities and an adverse pressure gradient (APG) boundary layer are calculated using the same numerical procedure as in $\mathrm{Hu}$ et al.. ${ }^{15}$ The results of one-point spectra, cross spectra and convective velocities for the wall pressure fluctuations are analyzed and compared to the experimental results from $\mathrm{Hu}$ and Herr. ${ }^{17}$ Firstly, the method is briefly described in section II. Secondly, the numerical setups and the flow conditions for the calculated cases are presented in section III. Finally, the results are discussed and compared to the experimental results in section IV.

\section{Numerical approach}

\section{II.A. Poisson equation}

Pressure fluctuations in an incompressible turbulent boundary layer are governed by a Poisson equation. For a turbulent boundary layer along a wall located at $x_{2}=0$ refer to Fig. 1, the Poisson equation resulting from a mean-flow in positive $x_{1}$-direction, becomes

$$
\Delta p=-\rho_{0}\left(2 \frac{\partial U_{1}}{\partial x_{2}} \frac{\partial u_{2}}{\partial x_{1}}+\frac{\partial^{2}}{\partial x_{i} \partial x_{j}}\left(u_{i} u_{j}-\overline{u_{i} u_{j}}\right)\right) .
$$

Here, $U_{1}$ denotes the mean-flow velocity component in $x_{1}$-direction and $u_{i}$ indicates velocity fluctuations; $\rho_{0}$ is the mean air density and $p$ is the fluctuating pressure. The source term on the right-hand side of Eq. (1) comprises two parts. The first part is the mean-shear turbulence interaction term and the second part is the turbulence-turbulence interaction term. If the boundary is a rigid flat surface, the fluctuating pressure can be calculated from the convolution of the free-space Green function of the Poisson equation with the right-hand side source term, i.e.,

$$
p(\mathbf{x}, t)=-\int_{\mathbf{V}_{\mathbf{s}}+\mathbf{V}_{\mathbf{s}}^{\prime}} \rho_{0}\left(2 \frac{\partial U_{1}}{\partial x_{2}} \frac{\partial u_{2}(\mathbf{y}, t)}{\partial x_{1}}+\frac{\partial^{2}}{\partial x_{i} \partial x_{j}}\left(u_{i} u_{j}-\overline{u_{i} u_{j}}\right)(\mathbf{y}, t)\right) \cdot g(\mathbf{x}-\mathbf{y}) d^{3} \mathbf{y} .
$$

In Eq. (2) the integration is carried out over the original source area $\mathbf{V}_{\mathbf{s}}$ plus a source area $\mathbf{V}_{\mathbf{s}}^{\prime}$ that represents an image of $\mathbf{V}_{\mathbf{s}}$ mirrored at the solid wall in order to realize the appropriate wall boundary condition $(\partial p / \partial n)_{x_{2}=0}=0$ of the pressure fluctuations. ${ }^{1}$

If we let $f(\mathbf{y}, t)$ represents the source terms, the convolution integral Eq. (2) can be expressed as

$$
\int_{\mathbf{V}_{\mathbf{s}}+\mathbf{V}_{\mathbf{s}}^{\prime}} f(\mathbf{y}, t) \cdot g(\mathbf{x}-\mathbf{y}) d^{3} \mathbf{y}=(f * g)(\mathbf{x}, t) .
$$

The equation is solved in wavenumber domain by using the convolution theorem:

$$
\mathcal{F}\{f * g\}(\mathbf{x}, t)=\mathcal{F}\{f(\mathbf{x}, t)\} \cdot \mathcal{F}\{g(\mathbf{x})\}=\hat{f}(\mathbf{k}, t) \cdot \hat{g}(\mathbf{k}) .
$$

Here $\hat{f}(\mathbf{k}, t)=\mathcal{F}\{f(\mathbf{x}, t)\}$ denotes the three-dimensional spatial Fourier transform from the consecutive application of the one-dimensional spatial Fourier transform for all spatial coordinates $x_{i}$. Consequently, a solution to the Poisson problem for pressure is obtained at a given time level by multiplying the spatial Fourier transform of the time-dependent source term with the Fourier transform of the free-space Green function and subsequently transforming back the result into physical space:

$$
p(\mathbf{x}, t)=\mathcal{F}^{-1}\{\hat{f}(\mathbf{k}, t) \cdot \hat{g}(\mathbf{k})\} .
$$

For an accurate numerical solution with this approach, a good approximation to the free-space Green function is required despite the artificial truncation and periodicity of the Green function represented on a finite computational domain. To circumvent this problem, a modification as introduced by Hockney and Eastwood ${ }^{16}$ is applied, which provides an exact realization of the free-space Green function in conjunction with a Fourier transform method on the finite domain. A detailed description of the Hockney's method applied to the Poisson problem is found in Hu et al.. ${ }^{15}$ 


\section{II.B. FRPM}

To calculate the fluctuating pressure an appropriate fluctuating velocity field within the turbulent boundary layer is needed. We use the Fast Random Particle-Mesh Method (FRPM) ${ }^{18}$ to generate synthetic turbulent velocity fluctuations to prescribe the right-hand side source term of Eq. (1). FRPM uses averaged turbulence statistics to synthesize the turbulent velocity fluctuations. One and two-point statistics are realized. The basic idea is to generate a fluctuating vector potential $\psi_{i}$ with three components from a convolution of spatial white noise $\mathcal{U}_{i}$ with a spatial Gaussian filter kernel $G$,

$$
\psi_{i}(\mathbf{x}, t)=\int_{\mathbf{V}_{\mathbf{s}}} \hat{A}(\mathbf{x}) G\left(\mathbf{x}-\mathbf{x}^{\prime}\right) \mathcal{U}_{i}\left(\mathbf{x}^{\prime}, t\right) d^{3} \mathbf{x}^{\prime},
$$

with

$$
G\left(\mathbf{x}-\mathbf{x}^{\prime}\right)=\exp \left(-\frac{\pi}{2} \frac{\left|\mathbf{x}-\mathbf{x}^{\prime}\right|}{l_{s}^{2}}\right),
$$

where $\hat{A}$ denotes an amplitude function whose appropriate scaling yields the desired variance of $\psi_{i}, \mathbf{x}$ defines field coordinates of the vector potential and $\mathbf{x}^{\prime}$ defines white noise field coordinates. Furthermore, $l_{s}$ is an integral turbulent length scale determined from Reynolds averaged Navier-Stokes (RANS).

The fluctuating velocities can be obtained by taking the curl of the fluctuating potential field $\boldsymbol{\psi}$,

$$
\mathbf{u}=\nabla \times \boldsymbol{\psi},
$$

or using tensor notation,

$$
u_{i}=\epsilon_{i j k} \frac{\partial \psi_{k}}{\partial x_{j}}
$$

For sufficiently slow spatially changing length scale $l_{s}$ and amplitude $\hat{A}$ the derivatives of $\psi_{i}$ can be expressed through analytical derivatives of the Gaussian filter kernel,

$$
u_{i}(\mathbf{x}, t)=\int_{\mathbf{V}_{\mathbf{s}}} \hat{A}(\mathbf{x}) \epsilon_{i j k} \frac{\partial G\left(\mathbf{x}-\mathbf{x}^{\prime}\right)}{\partial x_{j}} \mathcal{U}_{k}\left(\mathbf{x}^{\prime}, t\right) d^{3} \mathbf{x}^{\prime} .
$$

The white noise field $\mathcal{U}_{i}$ is defined in a Lagrangian frame moving at local flow velocity $\mathbf{U}$. Altogether, the properties of white noise are defined in a fixed Eulerian frame by

$$
\begin{aligned}
<\mathcal{U}_{i}\left(\mathbf{x}^{\prime}, t\right)> & =0, \\
<\mathcal{U}_{i}\left(\mathbf{x}^{\prime}, t\right) \mathcal{U}_{j}\left(\mathbf{x}^{\prime}+\mathbf{r}, t+\tau\right)> & =\delta(\mathbf{r}-\mathbf{U} \tau) \delta_{i j} .
\end{aligned}
$$

where the bracket means an ensemble average, $\delta_{i j}$ is the Kronecker symbol and $\delta(\mathbf{r}-\mathbf{U} \tau)$ describes a frozen turbulence flow moving with the flow velocity $\mathbf{U}$. Additional temporal turbulence decay can be modeled by a Langevin equation, which introduces the de-correlation in the two-points statistics. ${ }^{18}$ In the Eulerian frame a modification of the cross-correlation is given by

$$
<\mathcal{U}_{i}\left(\mathbf{x}^{\prime}, t\right) \mathcal{U}_{j}\left(\mathbf{x}^{\prime}+\mathbf{r}, t+\tau\right)>=\delta(\mathbf{r}-\mathbf{U} \tau) \exp \left(-\frac{|\tau|}{\tau_{s}}\right) \delta_{i j} .
$$

In this expression $\exp \left(-|\tau| / \tau_{s}\right)$ describes the turbulence decay, i.e. the spatially white noise is correlated in time with time-scale $\tau_{s}$. The local time scale is determined from RANS.

More details about the implementation in computational domain of FRPM and the one-point and two-point properties of the generated synthetic turbulence refer to the work. ${ }^{18,19}$

\section{Computational setups and mean flow conditions}

Mean flow statistics for the flat plate boundary layers are obtained from 2.5D RANS calculations, which are achieved by DLR's in-house CFD solver TAU with the Reynolds stress model. ${ }^{20}$ Both ZPG and APG flows are calculated. Fig. 1 shows a sketch of the computational domain. The length of the whole plate is $1370 \mathrm{~mm}$ with a leading edge length of $120 \mathrm{~mm}$. A $12^{\circ}$ beveled trailing edge on the underside of the plate is constructed to develop a ZPG boundary layer on the topside in the rear area. For the APG case, a NACA-0012 airfoil with a chord length of $400 \mathrm{~mm}$ is placed above the flat plate. The rotation axis is at $41 \%$ of the chord length. Identical geometries of the flat plate and the NACA airfoil to the experiment from $\mathrm{Hu}$ and $\mathrm{Herr}^{17}$ is used.

The wall fluctuating pressure beneath the boundary layer is calculated in a three-dimensional rectangular FRPM domain with its center located at $x_{1}=1170 \mathrm{~mm}$, see Fig. 1 . The dimension of the 
ZPG:

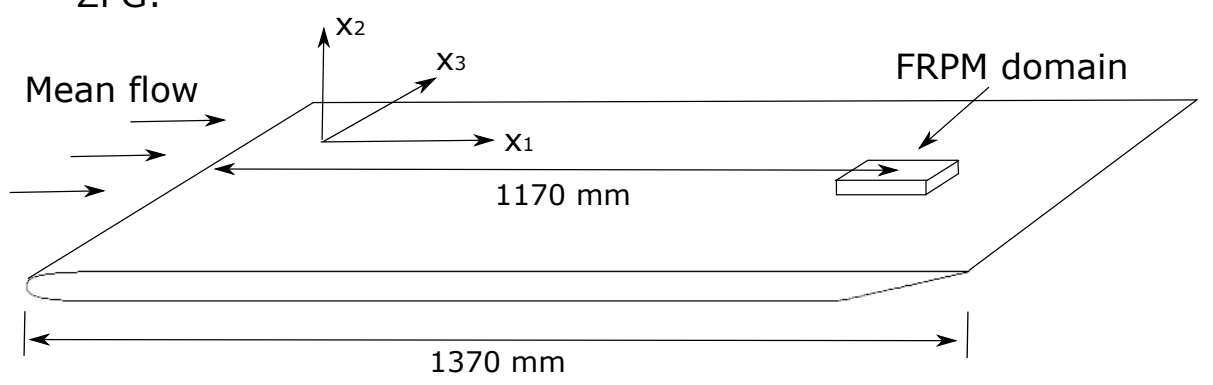

APG:

FRPM domain

Figure 1: Sketch of the computational domain for the ZPG flow (top) and the side view for the APG flow (down).

FRPM domain is $L_{1}=127 \mathrm{~mm}, L_{2}=21 \mathrm{~mm}$ and $L_{3}=63 \mathrm{~mm}$. Since the Hockney method demands a grid with $2^{N}$ mesh points in each direction, a cartesian grid with $128 \times 64 \times 64$ points is used in the calculation. The corresponding mesh size is $\Delta x_{1}, \Delta x_{3}=1 \mathrm{~mm}$ and $\Delta x_{2}=1 / 3 \mathrm{~mm}$. The calculated boundary layers share similar boundary layer thicknesses, therefore a same sized FRPM domain is used for all the calculation cases. The calculation time step is $\Delta t=1.67 \mathrm{e}-5 \mathrm{~s}$, i.e. the sampling rate is $60 \mathrm{kHz}$. Calculations for each case were carried on 4 CPUs and the computation time was about two weeks for calculating $1.5 \mathrm{~s}$ time series data.

Table 1: Comparison of the boundary layer parameters between the results from RANS and the experiment.

\begin{tabular}{ccccccc}
\hline \hline & $\begin{array}{c}U_{0} \\
(\mathrm{~m} / \mathrm{s})\end{array}$ & $\begin{array}{c}\delta \\
(\mathrm{mm})\end{array}$ & $\begin{array}{c}\delta^{*} \\
(\mathrm{~mm})\end{array}$ & $\begin{array}{c}\theta \\
(\mathrm{mm})\end{array}$ & $H$ & $R e_{\theta}=U_{0} \theta / \nu$ \\
\hline ZPG for two different & velocities, $x_{1}=1210 \mathrm{~mm}$ & \\
\hline RANS & 30.1 & 20.0 & 3.34 & 2.42 & 1.38 & 4737 \\
Experiment & 30.2 & 19.7 & 3.51 & 2.49 & 1.41 & 4889 \\
\hline RANS & 58.8 & 17.9 & 2.90 & 2.15 & 1.35 & 8230 \\
Experiment & 58.7 & 18.5 & 3.13 & 2.28 & 1.37 & 8685 \\
\hline \hline APG, $x_{1}=1128 \mathrm{~mm}$ & & & & & \\
\hline RANS & 32.9 & 15.6 & 3.32 & 2.09 & 1.59 & 4464 \\
Experiment & 32.0 & 23.0 & 5.09 & 3.12 & 1.63 & 6492 \\
\hline APG, $x_{1}=1210 \mathrm{~mm}$ & & & & & \\
\hline RANS & 30.4 & 19.7 & 5.05 & 2.93 & 1.72 & 5772 \\
Experiment & 30.4 & 28.7 & 7.68 & 4.39 & 1.75 & 8670 \\
\hline \hline
\end{tabular}

To generate the synthetic turbulence and the source terms for the Poisson equation, FRPM needs the mean flow statistics of the mean velocity profile $u$, kinetic energy $k$, length scale $l_{s}$ and the time scale $\tau_{s}$. The mean velocity profiles were measured at $x_{1}=1210 \mathrm{~mm}$ for ZPG and APG boundary layers and an additional point at $x_{1}=1128 \mathrm{~mm}$ for the APG boundary layer from Hu and Herr. ${ }^{17}$ The calculated mean flow velocity profiles from RANS are compared to the measured profiles, shown in Fig. 2. Results from RANS show good agreement to the measured results. Boundary layer parameters obtained from both RANS and the experiment are listed in table 1. Note that the boundary layer thickness for the APG boundary layer does not match the experimental results. The calculated boundary layer thickness is much thinner than the measured one. This is probably because the present RANS calculations do not take the open jet environment into account, which seems to be important for the APG case due 


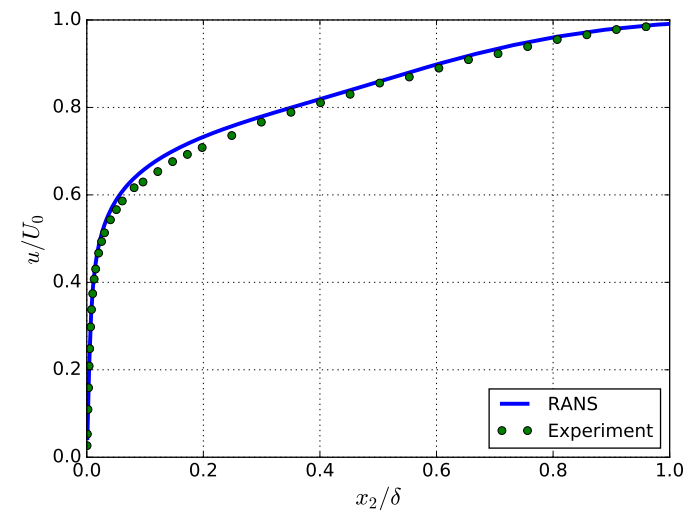

(a)

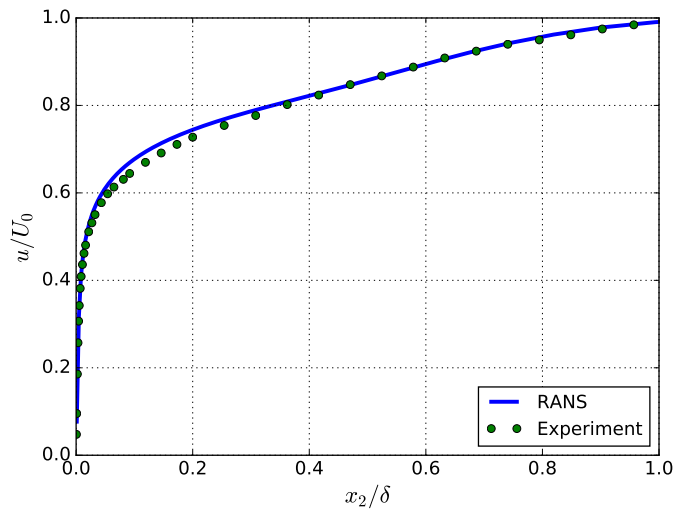

(b)

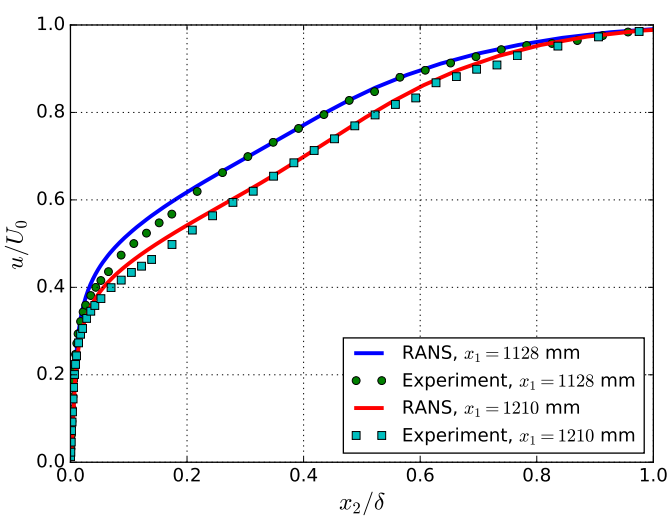

(c)

Figure 2: Comparison of the mean velocity profile between RANS and the experimental results; (a) ZPG for $30.1 \mathrm{~m} / \mathrm{s}$ at $x_{1}=1210 \mathrm{~mm}$; (b) ZPG for $58.8 \mathrm{~m} / \mathrm{s}$ at $x_{1}=1210 \mathrm{~mm}$; (c) APG at $x_{1}=1128 \mathrm{~mm}$ and $1210 \mathrm{~mm}$.

to the additional NACA airfoil. For the APG calculation case some changes to the airfoil position are made in order to obtain a similar boundary layer development between $1128<x_{1}<1210 \mathrm{~mm}$. In the calculation the airfoil is located $150 \mathrm{~mm}$ (chord rotation axis position) above the flat plate while in the measurement $120 \mathrm{~mm}$. The geometric angle of attack of the airfoil is $9^{\circ}$ in the calculation while $10^{\circ}$ in the measurement.

Comparisons of the kinetic energy between RANS and measurement results at $x_{1}=1210 \mathrm{~mm}$ are shown in Fig. 3. For the APG case the kinetic energy was only measured at $x_{1}=1210 \mathrm{~mm}$ in the experiment. In general, the results from RANS show good agreement to the measured data. However, a more rapid decrease trend in the outer region $x_{2}>0.5 \delta$ is shown. One reason to explain the trend could be the nearly zero turbulence intensity outside the boundary layer in the calculation domain. This forces the kinetic energy to approach zero at the boundary layer edge while in the measurement the freesteam flow has a larger turbulence intensity. The position of the maximum kinetic energy for the APG boundary layer is well predicted from RANS, however the level is larger compared to the measurement. This may be caused by the thinner boundary layer obtained from RANS.

The length scale $l_{s}$ and the time scale $\tau_{s}$ required by FRPM are determined from RANS, defined by

$$
\begin{aligned}
l_{s}^{*} & =\frac{c_{l}}{C_{\mu}} \frac{\sqrt{k^{*}}}{\omega^{*}}, \\
\tau_{s}^{*} & =\tau_{f} \frac{l_{s}^{*}}{\sqrt{k^{*}}} .
\end{aligned}
$$

where $k^{*}=k / U_{r e f}^{2}$ is the turbulent kinetic energy and $\omega^{*}=\omega \cdot l_{r e f} / U_{\text {ref }}$ is the specific rate of dissipation. The expression $*$ denotes non-dimensional parameters. The reference quantities are set to $U_{\text {ref }}=343 \mathrm{~m} / \mathrm{s}$ and $l_{\text {ref }}=1 \mathrm{~m}$. The constant $C_{\mu}=0.09$ and $c_{l}$ is estimated to be $0.54,{ }^{21}$ so the pre-factor $c_{l} / C_{\mu} \simeq 6.0$. 


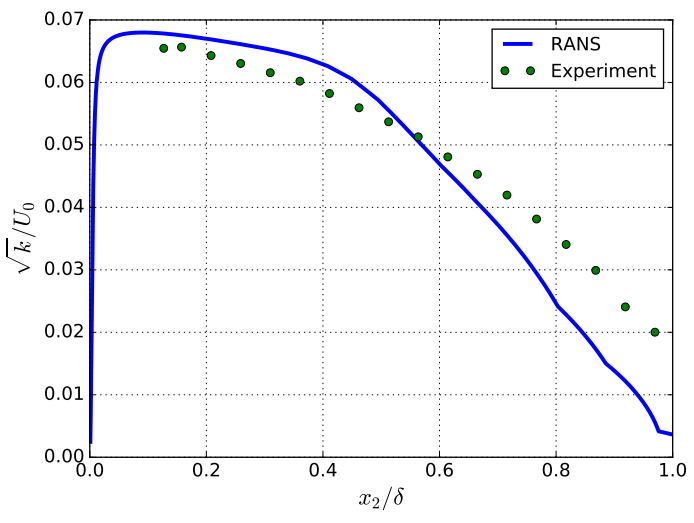

(a)

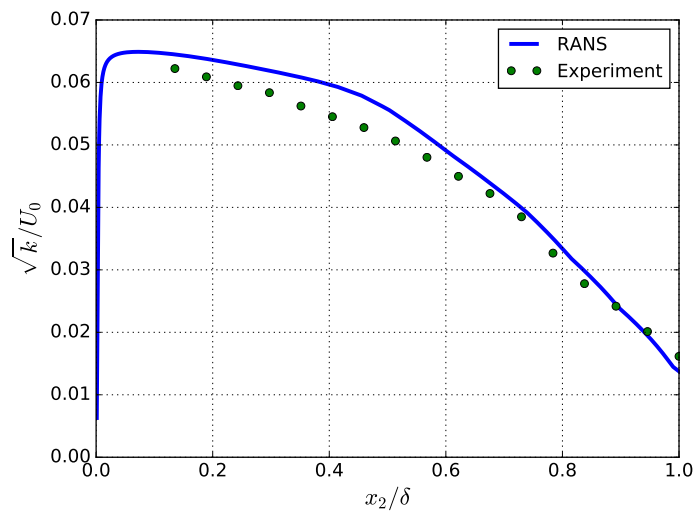

(b)

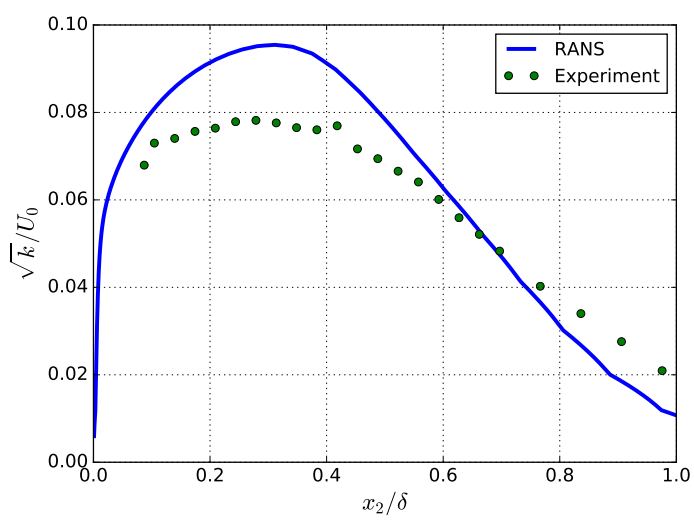

(c)

Figure 3: Comparison of the kinetic energy between RANS and experimental results at $x_{1}=1210 \mathrm{~mm}$; (a) ZPG for $30.1 \mathrm{~m} / \mathrm{s}$; (b) ZPG for $58.8 \mathrm{~m} / \mathrm{s}$; (c) APG.

The pre-factor of time scale $\tau_{f}$ is considered to be of order one. In this computation $\tau_{f}=0.7$ is used based on a good agreement of the longitudinal turbulence decay with the measured results.

\section{Results}

\section{IV.A. Turbulence velocity fluctuations realization}

Turbulence velocity fluctuations realized by FRPM are used to prescribe the fluctuating source terms of the Poisson equation (1) to derive unsteady surface pressure fluctuations. To verify a proper realization of fluctuating velocity from FRPM, Fig. 4 shows the reconstructed turbulent kinetic energy and the reconstructed variance of the velocity fluctuations in comparison to the target kinetic energy within the boundary layer. Through the isotropic turbulence approach the variance of the velocity fluctuations are nearly equally well realized for each component. In the outer region $>0.3 \delta$ a very good reconstruction of the kinetic energy is visible for all calculated cases. A lack of kinetic energy level is evident in the region $<0.3 \delta$, especially for $<0.1 \delta$. The reconstructed kinetic energy drops dramatically in the region $<0.1 \delta$, which is not the case in RANS. The reason for the loss in reconstructed energy is considered as follows. Firstly, in the inner region the kinetic energy and the length scale change fast which is not the optimal condition for FRPM kinetic energy realization, which assumes slowly changing turbulent intensities. Secondly, the grid resolution is not fine enough to resolve the small turbulence structures.

FRPM uses a spatial Gaussian filter to generate the fluctuating turbulence velocities, thus a Gaussian turbulence velocity spectrum is realized. The one-dimensional wavenumber spectra $\Phi_{i i}\left(k_{1}\right)$ realized by 


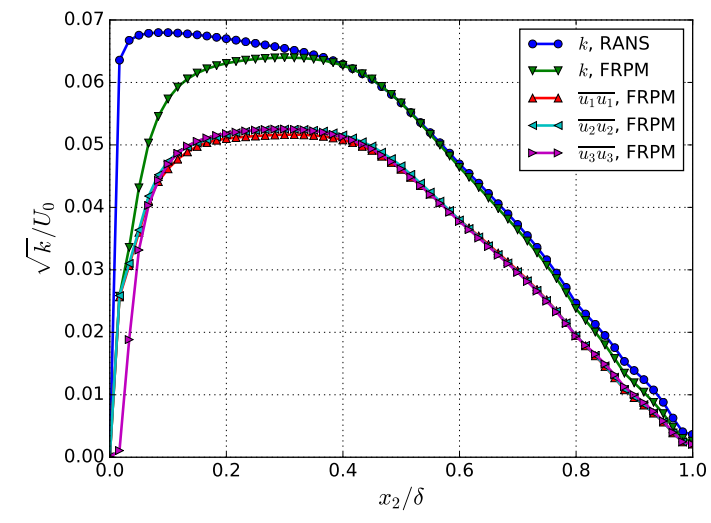

(a)

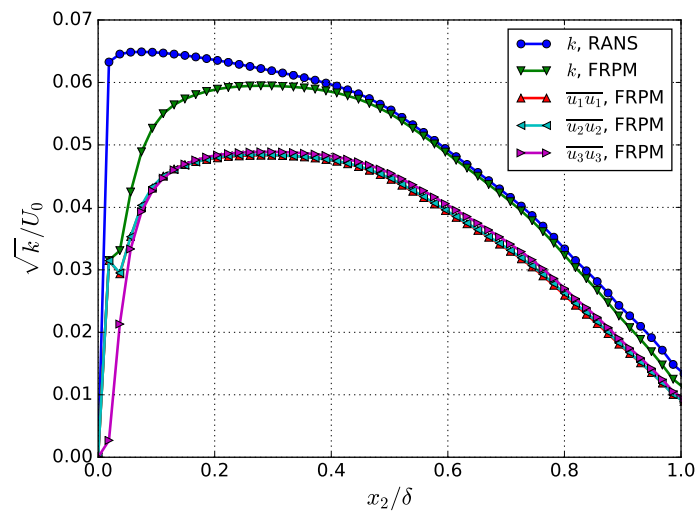

(b)

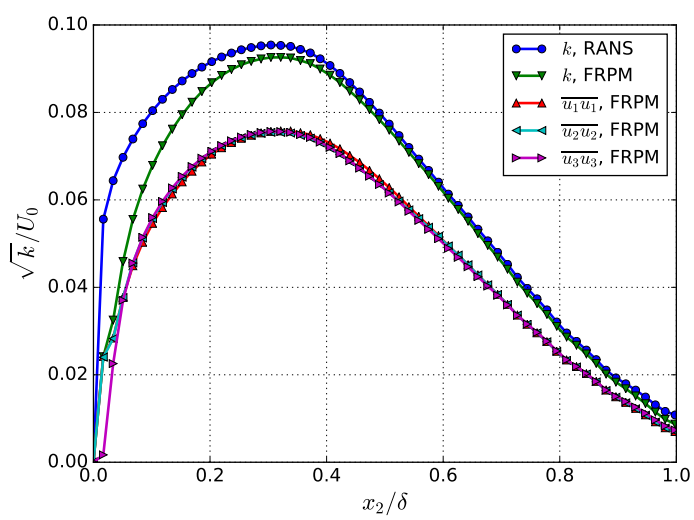

(c)

Figure 4: Reconstruction of the kinetic energy from FPRM in comparison to the target kinetic energy obtained from RANS; (a) ZPG for $30.1 \mathrm{~m} / \mathrm{s}$; (b) ZPG for $58.8 \mathrm{~m} / \mathrm{s}$; (c) APG.

FRPM can be expressed as ${ }^{15}$

$$
\begin{aligned}
\Phi_{11}\left(k_{1}\right) & =\frac{\overline{u_{1}^{2}} l_{s}}{\pi} \exp \left(\frac{-k_{1}^{2} l_{s}^{2}}{\pi}\right), \\
\Phi_{22}\left(k_{1}\right) & =\Phi_{33}\left(k_{1}\right)=\frac{\overline{u_{2,3}^{2}} l_{s}}{2 \pi^{2}} \exp \left(\frac{-k_{1}^{2} l_{s}^{2}}{\pi}\right)\left(2 k_{1}^{2} l_{s}^{2}+\pi\right) .
\end{aligned}
$$

Thus, the velocity spectra $\Phi_{i i}(\omega)$ can be obtained using the following relationship between the wavenumber spectra and the velocity spectra,

$$
\Phi_{i i}(\omega)=\int_{-\infty}^{\infty} \Phi_{i i}\left(k_{1}\right) \phi_{m}\left(\omega-k_{1} U_{c}\right) \mathrm{d} k_{1},
$$

where $\phi_{m}\left(\omega-U_{c} k_{1}\right)$ is the so-called moving-axis spectrum. The result for the velocity spectra depends on whether turbulence decay (finite decay time-scale $\tau_{s}$ ) is considered or if the assumption of frozen turbulence (Taylor's hypothesis) is applied by choosing $\tau_{s} \rightarrow \infty$. Thus, for a frozen turbulence $\phi_{m}\left(\omega-U_{c} k_{1}\right)=\delta\left(\omega-U_{c} k_{1}\right)$. Practically, considering an uniform mean flow in $x_{1}$-direction, the frequency spectrum of frozen turbulence is obtained by replacing wavenumber $k_{1}$ by $\omega / U_{c}$, where $U_{c}$ is the convection velocity in $x_{1}$-direction. For a non-frozen turbulence FRPM introduces a decay factor $\exp \left(-|\tau| / \tau_{s}\right)$ in Eq. (13) to describe the turbulence decay. The resulting $\phi_{m}\left(\omega-U_{c} k_{1}\right)$ can be analytically derived, follows ${ }^{15}$

$$
\phi_{m}\left(\omega-U_{c} k_{1}\right)=\frac{\tau_{s}}{\pi\left(1+\left(\omega-U_{c} k_{1}\right)^{2} \tau_{s}^{2}\right)} .
$$

For the wall pressure fluctuations, $\Phi_{22}(\omega)$ is the most important velocity spectra. The realized spectra for ZPG at velocity of $30.1 \mathrm{~m} / \mathrm{s}$ in comparison to the analytical frozen and non-frozen spectra based on 
the kinetic energy from RANS are shown in Fig. 5. An attenuation of the realized spectra is evident in the inner region, see Fig. 5(a). Especially for $x_{2}=0.03 \delta$ the level of the realized spectra is more than $10 \mathrm{~dB}$ less than the analytical Gaussian spectra. Besides the attenuation in level the roll-off of the realized spectrum for $x_{2}=0.03 \delta$ occurs at much lower frequencies compared to the analytical spectra. This is probably because the applied grid resolution is too rough to resolve the fine turbulence structure. In the outer region a very good agreement is obtained between the realized spectra and the analytical non-frozen spectra. Note that a less steep roll-off at higher frequencies for non-frozen turbulence is presented compared to frozen turbulence. The reason for this is that the introduced turbulence decay changes the form of the moving-axis spectrum $\phi_{m}\left(\omega-U_{c} k_{1}\right)$ from $\delta\left(\omega-U_{c} k_{1}\right)$ to a broadband form expressed in Eq. (19). As a consequence of an exchange of the $\delta$-function by a flattened distribution in the convolution integral Eq. (18), the energy of the frozen turbulence spectrum is spread out into adjacent frequency bands, in this case, from the lower frequencies into the higher frequencies. It is worth mentioning that the spectral contribution from turbulence decay depends on the form of $\phi_{m}$.

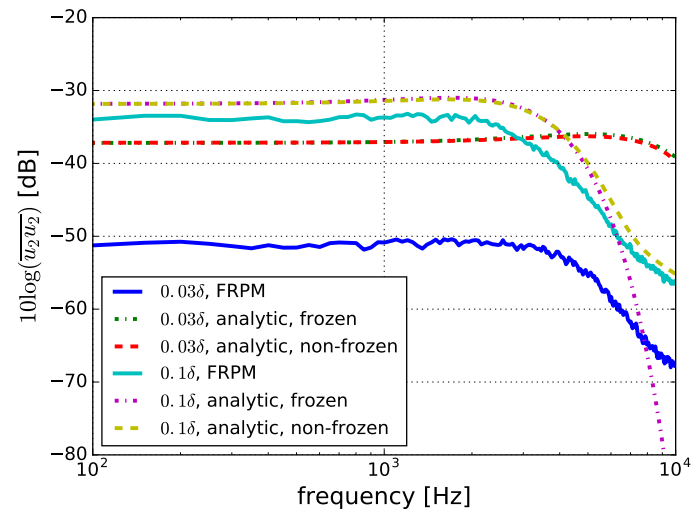

(a)

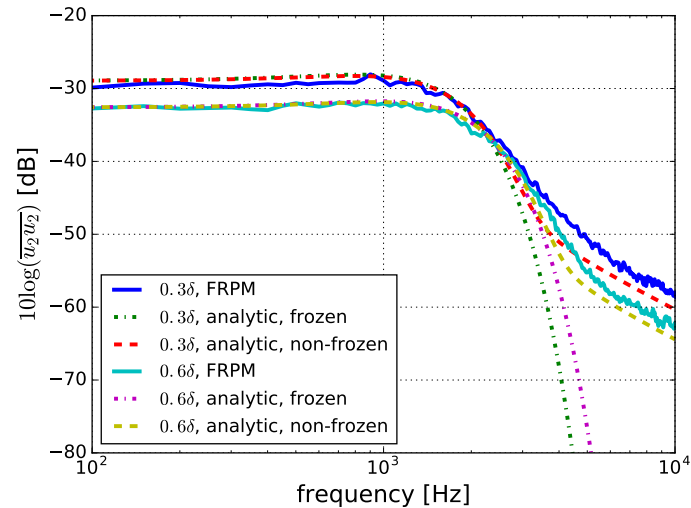

(b)

Figure 5: Realization of the velocity fluctuation spectra $\Phi_{22}$ for ZPG at velocity of $30.1 \mathrm{~m} / \mathrm{s}$ in comparison to the analytical frozen and non-frozen spectra based on the kinetic energy from RANS; (a) the inner region $x_{2}=0.03 \delta$ and $0.1 \delta$; (b) the outer region $x_{2}=0.3 \delta$ and $0.6 \delta$.

\section{IV.B. One-point spectra}

The source term of the Poisson equation (1) comprises two contributions, the mean-shear turbulence interaction term and the turbulence-turbulence interaction term. Since the Poisson equation is linear, the solution of pressure fluctuations can be separately solved,

$$
\begin{aligned}
p_{m s}(\mathbf{x}, t) & =-2 \rho_{0} \int_{\mathbf{V}_{\mathbf{s}}+\mathbf{V}_{\mathbf{s}}^{\prime}} \frac{\partial U_{1}}{\partial x_{2}} \frac{\partial u_{2}(\mathbf{y}, t)}{\partial x_{1}} \cdot g(\mathbf{x}-\mathbf{y}) d^{3} \mathbf{y} \\
p_{t t}(\mathbf{x}, t) & =-\rho_{0} \int_{\mathbf{V}_{\mathbf{s}}+\mathbf{V}_{\mathbf{s}}^{\prime}} \frac{\partial^{2}}{\partial x_{i} \partial x_{j}}\left(u_{i} u_{j}-\overline{u_{i} u_{j}}\right)(\mathbf{y}, t) \cdot g(\mathbf{x}-\mathbf{y}) d^{3} \mathbf{y},
\end{aligned}
$$

where $p_{m s}$ denotes the pressure fluctuations for the mean-shear term and $p_{t t}$ for the turbulence-turbulence term. Realized variance is used as input values for $\overline{u_{i} u_{j}}$ by $i=j$, and set $\overline{u_{i} u_{j}}=0$ by $i \neq j$, since the flow is realized as an isotropic turbulence flow. The integrals of Eq. (20-21) can be performed by different approaches. This issue was discussed in the work of $\mathrm{Hu}$ et al.. ${ }^{15}$ It was found that the approach taking integration by parts for both source terms gives the best numerical solution. Thus, the expression for the total pressure fluctuations $p_{\text {total }}$ summed by both parts is given by

$$
\begin{gathered}
p_{\text {total }}(\mathbf{x}, t)=p_{m s}(\mathbf{x}, t)+p_{t t}(\mathbf{x}, t) \\
=\rho_{o} \int_{\mathbf{V}_{\mathbf{s}}+\mathbf{V}_{\mathbf{s}}^{\prime}}\left\{2 \frac{\partial U_{1}}{\partial x_{2}} u_{2}(\mathbf{y}, t) \cdot \frac{\partial g(\mathbf{x}-\mathbf{y})}{\partial x_{1}}+\frac{\partial}{\partial x_{i}}\left(u_{i} u_{j}-\overline{u_{i} u_{j}}\right)(\mathbf{y}, t) \cdot \frac{\partial g(\mathbf{x}-\mathbf{y})}{\partial x_{j}}\right\} d^{3} \mathbf{y} .
\end{gathered}
$$

Figure 6 shows the simulated one-point spectra of $p_{m s}, p_{t t}$ and $p_{\text {total }}$. The results clearly show the dominance of $p_{m s}$ for the wall pressure fluctuations. The level of $p_{m s}$ is larger than $p_{t t}$ over the whole frequency range for all calculated cases. In contrast to $p_{m s}$ which has a maximum at mid frequencies, $p_{t t}$ shows a low-pass behaviour with a maximum plateau at low frequencies. The maximum difference 


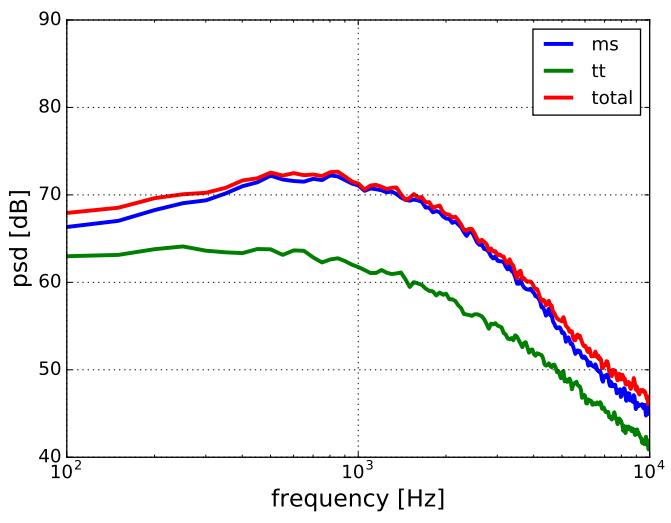

(a)

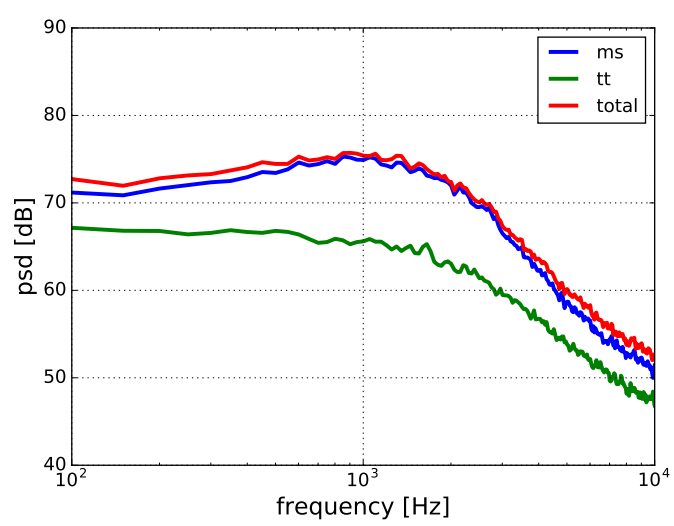

(c)

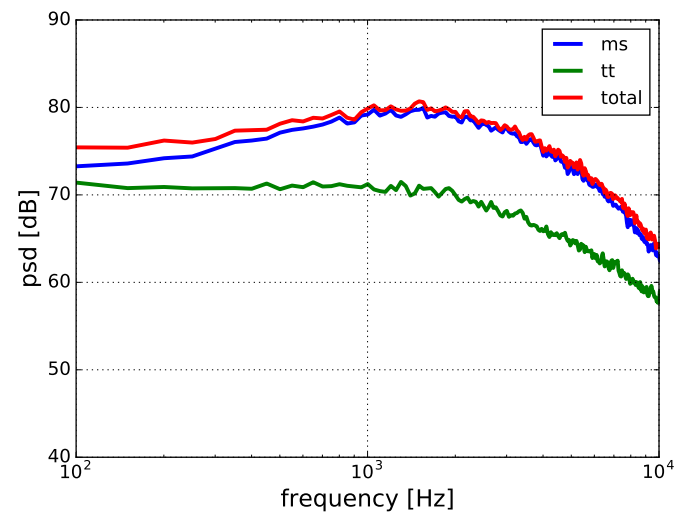

(b)

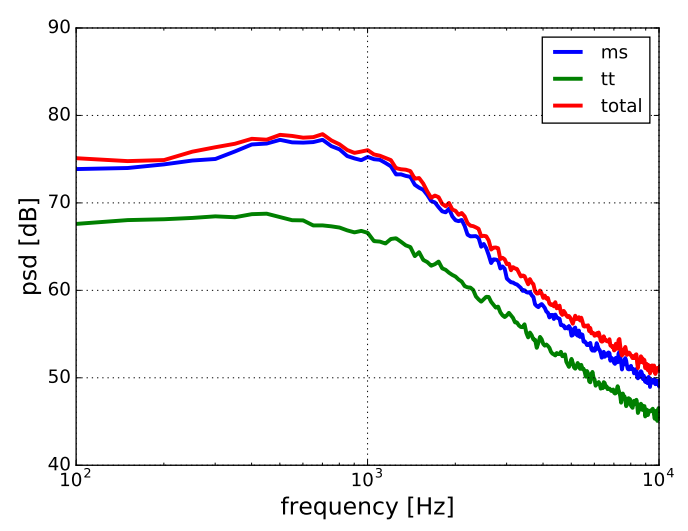

(d)

Figure 6: One-point spectra of $p_{m s}, p_{t t}$ and $p_{\text {total }}$; (a) ZPG for $30.1 \mathrm{~m} / \mathrm{s}$; (b) ZPG for $58.8 \mathrm{~m} / \mathrm{s}$; (c) APG $x_{1}=1128 \mathrm{~mm}$; (d) APG $x_{1}=1210 \mathrm{~mm}$.

between $p_{m s}$ and $p_{t t}$ is about $10 \mathrm{~dB}$ at mid frequencies where $p_{m s}$ has the maximum level. At lower frequencies the difference becomes smaller, e.g. only about $2 \mathrm{~dB}$ at $100 \mathrm{~Hz}$ for ZPG velocity of $58.8 \mathrm{~m} / \mathrm{s}$.

The simulated spectra of $p_{\text {total }}$ are compared to the experimental results, shown in Fig. 7. The low frequency trend and the maximum level of the simulated spectra are consistent with the measured spectra. However, the simulated spectra drop too fast at high frequencies. This is primarily due to the lack of the reconstructed kinetic energy in the near wall region $<0.1 \delta$, which contributes mostly to high frequencies. Note that the boundary layer thickness for the simulated APG boundary layer is much thinner than the measured case, this causes a spectral shift to higher frequencies. In any case, the development of the APG spectra which shift to lower frequencies in downstream direction is well represented.

Alternatively, the wall pressure spectrum of $p_{m s}$ defined by $\Phi_{p p m s}(\omega)$ can be analytically solved for statistically stationary turbulence using the mean flow one-point statistics together with the two-point cross-correlation model that underlies the synthetic turbulence generation. The solution of Eq. (20) follows $^{1}$

$$
\begin{aligned}
\Phi_{\text {ppms }}(\omega)= & 4 \rho_{0}^{2} \int_{0}^{\delta} \int_{-\infty}^{\infty} \int_{-\infty}^{\infty} \frac{k_{1}^{2}}{k^{2}} \exp \left(-2 k x_{2}\right) \overline{u_{2}^{2}}\left(x_{2}\right) \frac{\partial U_{1}^{2}\left(x_{2}\right)}{\partial x_{2}} \Lambda_{2}\left(x_{2}\right) \\
& \Phi_{22}\left(k_{1}, k_{3}, x_{2}\right) \phi_{m}\left(\omega-k_{1} U_{c}\left(x_{2}\right)\right) \mathrm{d} k_{1} \mathrm{~d} k_{3} \mathrm{~d} x_{2} .
\end{aligned}
$$

where $k_{i}$ is wavenumber component in each direction and $k^{2}=k_{1}^{2}+k_{3}^{2}$. The quantity of $\overline{u_{2}^{2}}\left(x_{2}\right)$ is the turbulence variance in wall-normal direction, $U_{1}\left(x_{2}\right)$ is the local flow velocity, and $U_{c}\left(x_{2}\right)$ is the local flow convective velocity. For the present problem with convection in $x_{1}$-direction and assumed statistical stationarity in $x_{1}, U_{c}\left(x_{2}\right)$ can be calculated from the maximum of the cross-correlation function $R_{u u}\left(\Delta x_{1}, 0, \tau\right)$. The convective velocity follows from $U_{c}=\Delta x_{1} / \tau\left(\Delta x_{1}\right)$, where $\tau$ is the time shift for 


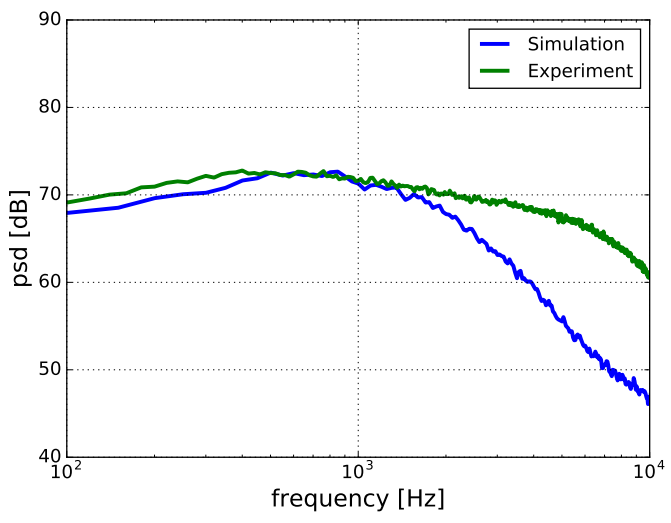

(a)

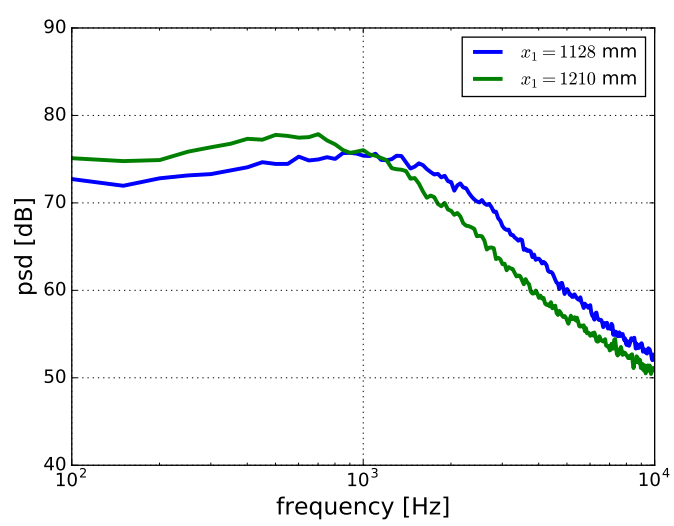

(c)

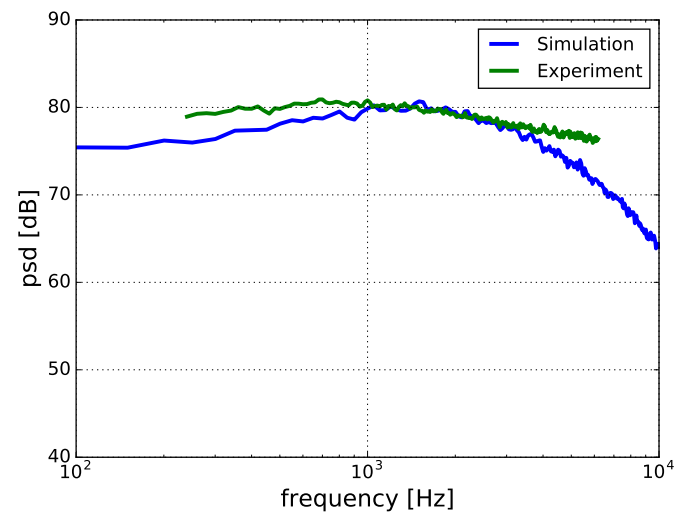

(b)

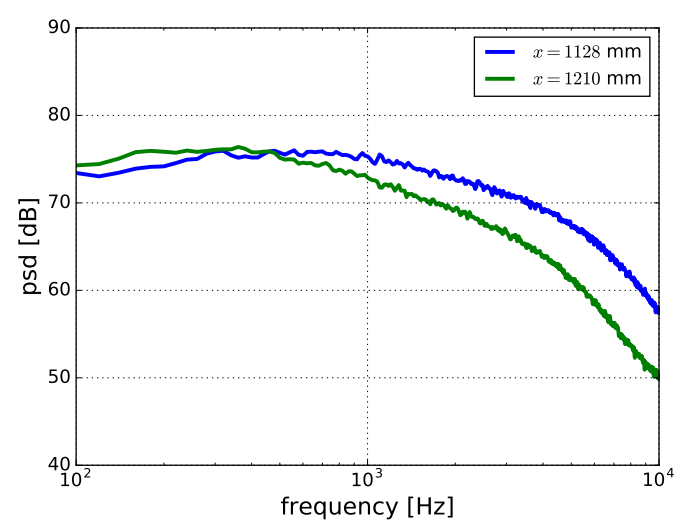

(d)

Figure 7: Comparison of the one-point spectra; (a) ZPG for $30.1 \mathrm{~m} / \mathrm{s}$; (b) ZPG for $58.5 \mathrm{~m} / \mathrm{s}$; (c) APG, simulated spectra; (d) APG, measured spectra.

the velocity fluctuations at which $R_{u u}$ is maximal, and $\Delta x_{1}$ indicates the two-point separation in $x_{1^{-}}$ direction. The local convective velocity $U_{c}\left(x_{2}\right)$ is obtained using the simulation data. The results indicate $U_{c}\left(x_{2}\right)=U_{1}\left(x_{2}\right)$ across the boundary layer. The same results were reported by Wooldridge and Willmarth ${ }^{22}$ through measurements of correlation between the velocity fluctuations and the wall pressure fluctuations. $\Lambda_{2}\left(x_{2}\right)$ is the double-sided correlation length in wall-normal direction, i.e. $\Lambda_{2}\left(x_{2}\right)=2 l_{s}\left(x_{2}\right)$, where $l_{s}$ is the integral length scale used in FRPM. The function $\phi_{m}\left(\omega-k_{1} U_{c}\left(x_{2}\right)\right)$ is the moving axis spectrum which is introduced in FRPM through the turbulence decay term $\exp \left(-|\tau| / \tau_{s}\right)$, refer to Eq. (19). The velocity wavenumber spectrum of the vertical fluctuating velocity $\Phi_{22}\left(k_{1}, k_{3}\right)$ can be obtained by $\int \Phi_{22}\left(k_{1}, k_{2}, k_{3}\right) \mathrm{d} k_{2}$ and is given for the realized Gaussian spectra $\operatorname{as}^{15}$

$$
\Phi_{22}\left(k_{1}, k_{3}\right)=\frac{l_{s}^{4}\left(k_{1}^{2}+k_{3}^{2}\right) \exp \left(-l_{s}^{2}\left(k_{1}^{2}+k_{3}^{2}\right) / \pi\right)}{\pi^{3}} .
$$

Fig. 8 shows a very good agreement between the theoretical prediction for the non-frozen turbulence and the simulated spectrum for ZPG of $30.1 \mathrm{~m} / \mathrm{s}$. The discrepancy at high frequencies is probably caused by the attenuation of the realized turbulence due to the numerical grid resolution. Eq. (23) provides the wall-pressure spectrum as an integral over all decks of the boundary layer. The theoretical contributions to the wall pressure fluctuations from different wall-normal positions are also presented in Fig. 8. It shows that positions closer to the wall are responsible for the higher frequencies while both the inner and the outer region contribute to the lower frequencies. However, the position far away from the wall e.g. $x_{2}=0.6 \delta$ shows only little relevance to the wall pressure fluctuations. Due to the term $k_{1}^{2} / k^{2} \exp \left(-2 k x_{2}\right)$ in Eq. (23) the contribution of different wall-normal positions to the wall pressure spectra vanishes especially at lower and higher frequencies. For example the maximum contribution of the wall-normal position $x_{2}=0.1 \delta$ is located between $1-2 \mathrm{kHz}$. It is found that the frequency range of the maximum occurs in the plateau region of the velocity fluctuation spectra $\Phi_{22}$, see Fig. 5(a). This indicates that the energy-containing range of the spectra dominates the contribution to the wall pressure 
fluctuations, and the inertial subrange, which can not be well represented by the realized Gauss spectra due to the rapid roll-off at higher frequencies, may only play a minor role.

Furthermore, the contributions of different wall-normal positions for non-frozen turbulence show larger level at lower and higher frequencies compared to frozen turbulence. This is due to the turbulence decay, which spreads the energy out into the neighborhood bands. Consequently, the $\omega^{2}$ behavior for the wall pressure spectra at low frequencies, based on an assumption of frozen turbulence, disappears. Also the importance of the near wall region to the wall pressure fluctuations at lower frequencies increases. Note that the level of the realized velocity fluctuation spectra $\Phi_{22}$ at $x_{2}=0.03 \delta$ is more than $10 \mathrm{~dB}$ smaller than the analytical spectra calculated by using the kinetic energy from RANS, see Fig. 5(a). This attenuation in the realized velocity fluctuation spectra in the near wall region takes direct responsibility for the attenuation of the simulated wall pressure spectra at high frequencies shown in Fig. 7.

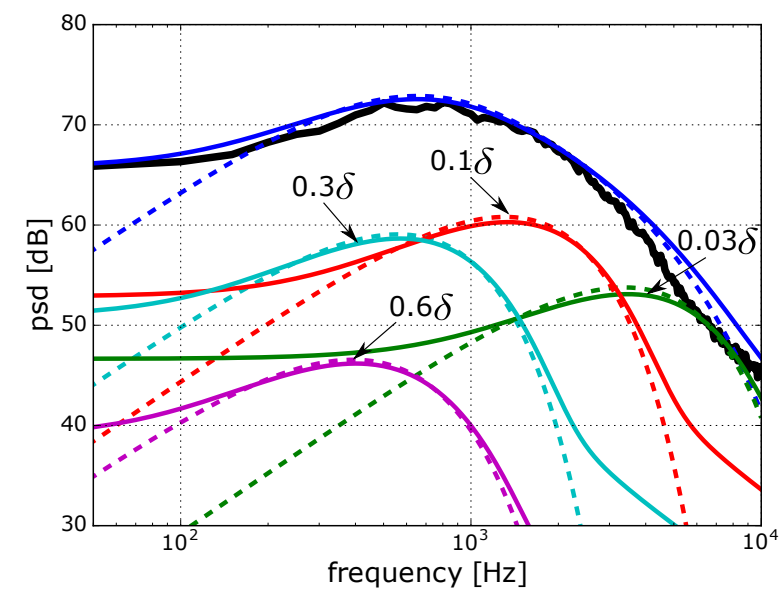

(a)

Figure 8: Spectra of $p_{m s}$ and the contributions to $p_{m s}$ from different wall-normal positions at $x_{2}=0.03 \delta$, $0.1 \delta, 0.3 \delta$ and $0.6 \delta$ for ZPG of $30.1 \mathrm{~m} / \mathrm{s}$; (-), numerical result; (-), theoretical results for non-frozen turbulence; (- -), theoretical results for frozen turbulence.

\section{IV.C. Cross spectra and convective features}

The spatial and temporal features of the wall pressure fluctuations can be studied from the two-point statistics. The time-space correlation of the pressure fluctuations is defined by

$$
R_{p p}(\mathbf{x}, \mathbf{r}, \tau)=<p(\mathbf{x}, t) p(\mathbf{x}+\mathbf{r}, t+\tau)>.
$$

For a slowly growing boundary layer, e.g. the calculated domain of the ZPG boundary layers, the flow field can be treated as a homogeneous field. Thus, $R_{p p}(\mathbf{x}, \mathbf{r}, \tau) \simeq R_{p p}(\mathbf{r}, \tau)$. Cross spectra can be calculated by taking Fourier transform of $\tau$ for the time-space correlation $R_{p p}(\mathbf{r}, \tau)$, as follows

$$
\Phi_{p p}(\mathbf{r}, \omega)=\frac{1}{2 \pi} \int_{-\infty}^{\infty} R_{p p}(\mathbf{r}, \tau) \exp (-i \omega \tau) \mathrm{d} \tau .
$$

If $\mathbf{r}=0$, we obtain the one-point spectrum $\Phi_{p p}(\omega)$. The coherence spectrum is defined by

$$
\Gamma(\mathbf{r}, \omega)=\frac{\left|\Phi_{p p}(\mathbf{r}, \omega)\right|}{\sqrt{\Phi_{p p}(\mathbf{0}, \omega)} \sqrt{\Phi_{p p}(\mathbf{r}, \omega)}} .
$$

The coherence spectra from different separations in longitudinal, $\mathbf{r}=r_{1} \cdot \mathbf{e}_{1}$, and the lateral coherence, $\mathbf{r}=r_{3} \cdot \mathbf{e}_{3}$, of the wall pressure fluctuations collapse at mid and high frequencies. At low frequencies the similarity behaviour loses and the coherence drops. Corcos ${ }^{3}$ used exponential functions to characterise the features of the cross spectra by taking advantage of the similarity of the turbulence decay, reads

$$
\left|\Gamma\left(r_{1}, r_{3}, \omega\right)\right|=\exp \left(-\alpha \omega r_{1} / U_{c}\right) \exp \left(-\beta \omega r_{3} / U_{c}\right),
$$

where $U_{c}$ is the convective phase velocity, $\alpha$ and $\beta$ are empirical constants which are in charge of prescribing the turbulence decay in longitudinal and lateral direction, respectively. A larger value of the 


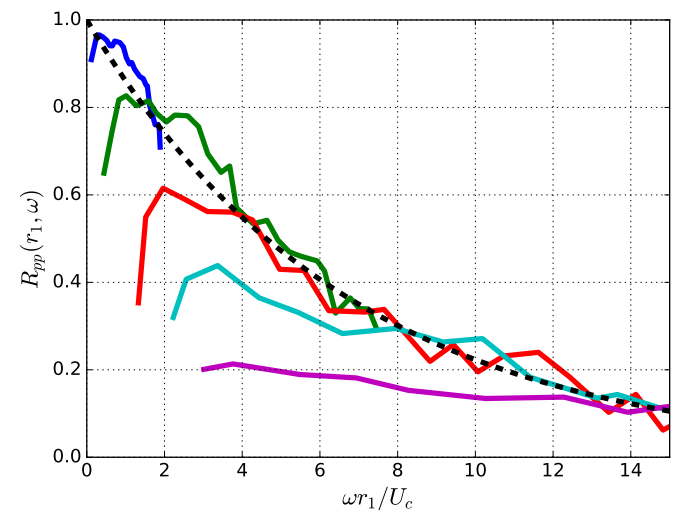

(a)

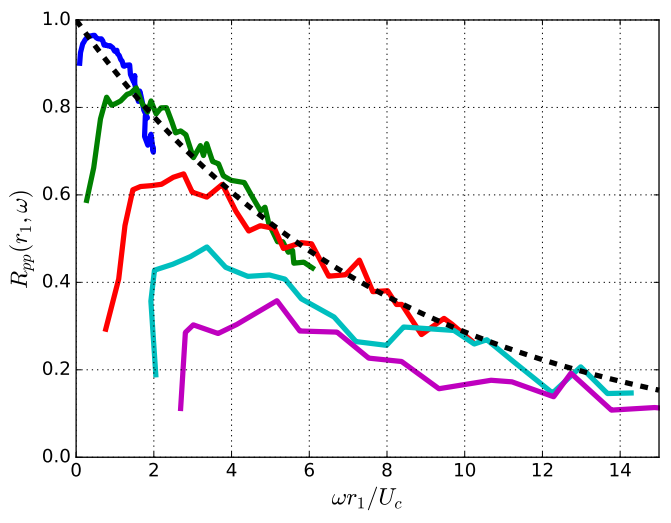

(b)

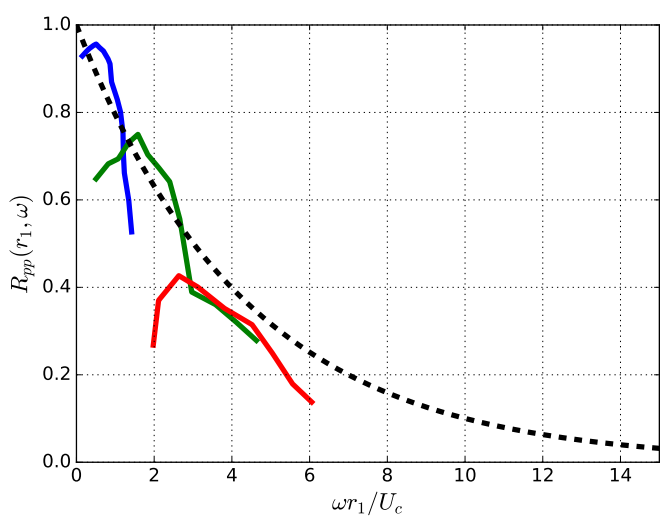

(c)

Figure 9: Comparison of the longitudinal coherence, (-), simulation; (- -), experiment; (a) ZPG for $30.1 \mathrm{~m} / \mathrm{s}, 0.6 \delta^{*}<r_{1}<12.6 \delta^{*} ;(--), \exp \left(-0.15 \omega r_{1} / U_{c}\right)$; (b) ZPG for $58.5 \mathrm{~m} / \mathrm{s}, 0.7 \delta^{*}<r_{1}<14.5 \delta^{*}$; $(--), \exp \left(-0.125 \omega r_{1} / U_{c}\right)$; (c) APG, $0.4 \delta^{*}<r_{1}<3.0 \delta^{*} ;(--), \exp \left(-0.23 \omega r_{1} / U_{c}\right)$.

constants indicates a more rapid decay of the cross spectra for the wall pressure fluctuations. Hu and Herr ${ }^{17}$ measured $\alpha=0.15$ and 0.125 for the ZPG boundary layers at velocities of $30.2 \mathrm{~m} / \mathrm{s}$ and $58.7 \mathrm{~m} / \mathrm{s}$, respectively. Generally, the value of $\alpha$ depends on Reynolds number and a larger Reynolds number results in a smaller value. A value of $\beta=0.72$ was measured for both velocities. For the APG boundary layer the coherence spectra can not be well characterised with a single exponential curve. However, as convenience for the comparison an exponential function for both longitudinal and lateral direction is drawn from the experimental results. The obtained value of the constants follows $\alpha=0.23$ and $\beta=0.55$.

Figs. 9-10 show the longitudinal and lateral coherence of $p_{\text {total }}$ as a function of $\omega r_{1,3} / U_{c}$. The convective phase velocity $U_{c}$ is defined by $U_{c}\left(r_{1}, \omega\right)=r_{1} \omega / \theta\left(r_{1}, \omega\right)$, where $\theta\left(r_{1}, \omega\right)$ is the phase difference of $\Gamma\left(r_{1}, 0, \omega\right)$. To present the coherence with a separation of $r_{1}$ in longitudinal direction, a phase velocity $U_{c}\left(r_{1}, \omega\right)$ obtained at the same longitudinal separation is used. The phase velocity does not depend on the lateral separations. A single phase velocity $U_{c}(\omega)$ obtained by the closest virtual microphones $r_{1}=2 \mathrm{~mm}$ is used for plotting the lateral coherence. The obtained exponential curves from the experiment for prescribing the coherence are also plotted for comparison.

The simulated longitudinal coherence shows very good agreement with the experimental results, see Fig. 9. The decay of the coherence is much stronger for the APG boundary layer than the ZPG boundary layers. Furthermore, a slightly slower decay for the higher velocity of the ZPG boundary layers is also presented in the simulation.

A less good agreement between the simulation and experimental results for the lateral coherence is shown in Fig. 10. The measured curves show much stronger decays than the simulated lateral coherence. This is probably due to the applied isotropic turbulence in FRPM while in real boundary layer flow the turbulence is stretched in longitudinal direction. Thus, the lateral correlation in the simulation extends over a longer distance and consequently results a slower decay in coherence.

Fig. 11 shows the comparison of the convective phase velocity for ZPG at velocity of $30.1 \mathrm{~m} / \mathrm{s}$. 


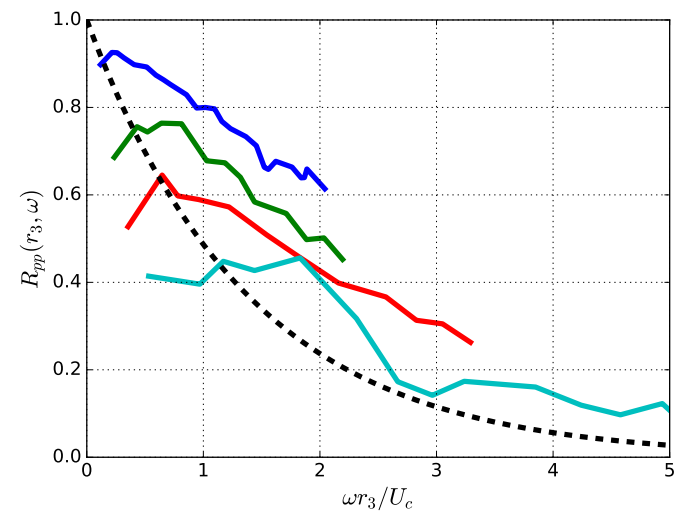

(a)

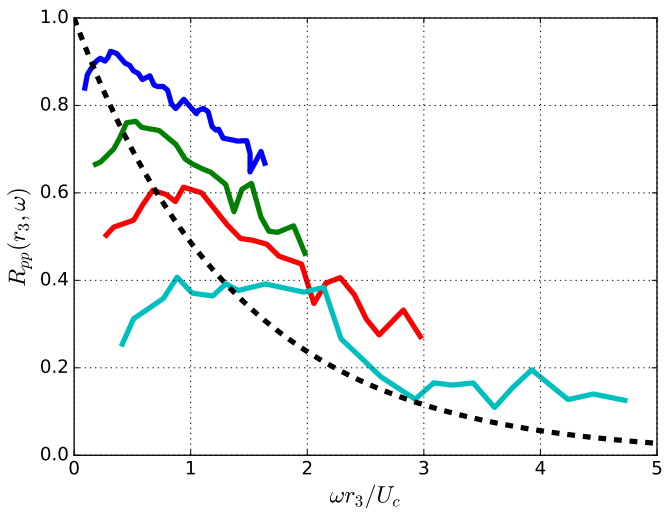

(b)

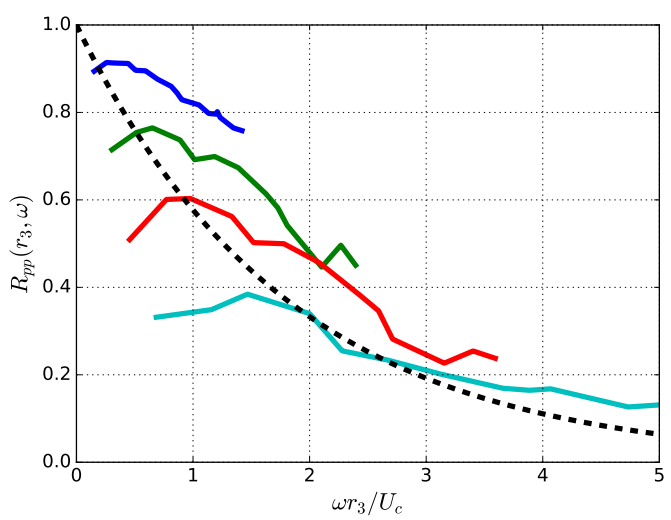

(c)

Figure 10: Comparison of the lateral coherence, (-), simulation; (- -), experiment; (a) ZPG for $30.1 \mathrm{~m} / \mathrm{s}, 0.6 \delta^{*}<r_{3}<2.7 \delta^{*} ;(--), \exp \left(-0.72 \omega r_{3} / U_{c}\right)$; (b) ZPG for $58.5 \mathrm{~m} / \mathrm{s}, 0.7 \delta^{*}<r_{3}<3.1 \delta^{*}$; $(--), \exp \left(-0.72 \omega r_{3} / U_{c}\right) ;(\mathrm{c}) \mathrm{APG}, 0.4 \delta^{*}<r_{3}<1.8 \delta^{*} ;(--), \exp \left(-0.55 \omega r_{3} / U_{c}\right)$.

The simulated velocity of $p_{\text {total }}$ increases at low frequencies, after reaching the maximum it decreases gradually with increasing frequencies. The maximum velocity is about $0.8 U_{0}$ and located between $0.2-$ $0.3 \omega \delta / U_{0}$. The frequency behaviour and the obtained maximum of the simulated phase velocity show good agreement with the measured results. However, the measured results present an increasing velocity with a larger longitudinal distance. The reason is that the eddies closer to the wall move with a slower velocity and die out over a shorter distance. They contribute to the wall pressure fluctuations not only at high frequencies but also at low frequencies. Thus, a slower velocity over a broadband frequency range was measured at a closer distance. However, this is not or only poorly presented in the simulation results because of the lack of the realized kinetic energy from FRPM in the near wall region.

Fig. 12 shows the comparison of the mean convective velocity $\overline{U_{c}}$, which is obtained by using the time shift $\tau$ of the maximum time-space correlation $R_{p p}\left(r_{1}, \tau\right)$ for a fixed longitudinal separation $r_{1}$, $\bar{U}_{c}\left(r_{1}\right)=r_{1} / \tau\left(r_{1}\right)$. Results for the maximum correlation smaller than 0.03 are not considered. Curves presenting the experimental results are drawn by best fit of the measured data. Simulated mean velocities of $p_{\text {total }}$ show similar trends to the measured results. The velocity increases at larger distances and is larger for the ZPG boundary layers than the APG. However, the measured velocities at closer distances are smaller than the simulated results. This is due to the lack of the realized kinetic energy from FRPM in the computation as discussed before.

\section{Conclusion}

Wall pressure fluctuations beneath ZPG and APG turbulent boundary layers are simulated with an effective numerical procedure. A Poisson equation is solved in the wavenumber domain using Hockney's method. The source terms including the mean-shear term and the turbulence-turbulence term on the right-hand side of the equation are realized using synthetic turbulence generated by FRPM. The turbu- 


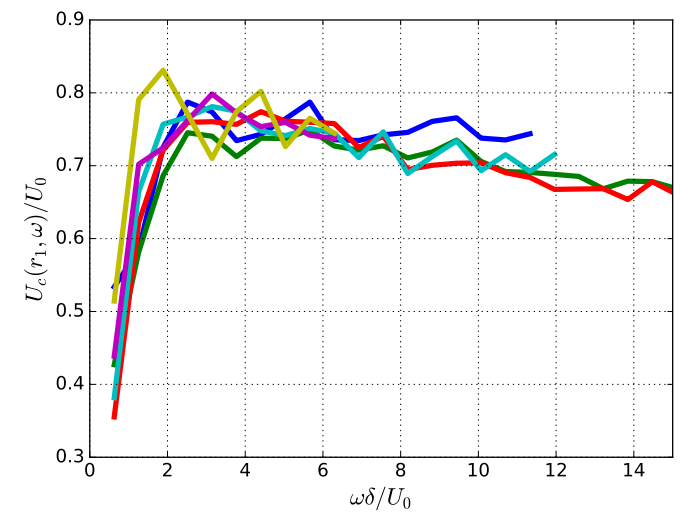

(a)

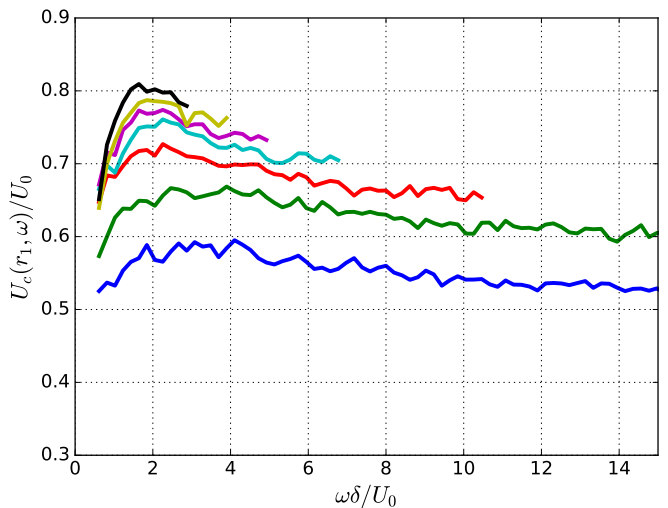

(b)

Figure 11: Comparison of the convective phase velocity for ZPG at velocity of $30.1 \mathrm{~m} / \mathrm{s}$; (a) simulation results, $0.6 \delta^{*}<r_{1}<18.0 \delta^{*}$; (b) experimental results, $0.6 \delta^{*}<r_{1}<23.4 \delta^{*}$.

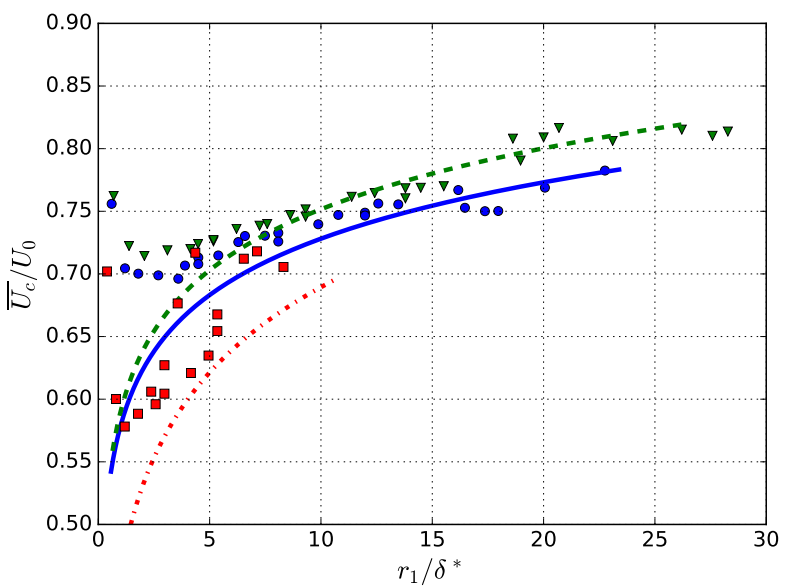

(a)

Figure 12: Comparison of the mean convective velocity. ZPG for $30.1 \mathrm{~m} / \mathrm{s}: \bullet$, simulation, (-), experiment; ZPG for $58.8 \mathrm{~m} / \mathrm{s}: \mathbf{\nabla}$, simulation, (- -), experiment; APG: $\mathbf{}$, simulation, (-.-), experiment.

lence velocity fluctuations are well realized except for the near wall region, especially for $<0.1 \delta$, where a lack of reconstructed kinetic energy is evident compared to the kinetic energy provided by RANS computations.

The resulting one-point spectra, cross spectra and the convective velocities of the wall pressure fluctuations are compared to the experimental results. The levels and the trends at lower frequencies of the one-point spectra are well predicted. An attenuation at higher frequencies is presented which is primarily due to the lack of the realized kinetic energy in the near wall region. This also causes a larger convective velocity at closer longitudinal distances because of the loss of the contributions of eddies closer to the wall, which move slower and die out faster. The simulated one-point spectrum contributed from the mean-shear part is compared to the theoretical prediction based on the flow parameters provided by FRPM. A very good agreement between both results is obtained. Furthermore, effects of turbulence decay on the one-point spectra is illustrated. The $\omega^{2}$ spectral behavior at low frequencies disappears when a non-frozen turbulence flow is considered. The major features of the cross spectra and the convective velocities are well determined and consistent with the measured results. A smaller coherence decay in lateral direction is presented probably due to the applied isotropic turbulence.

\section{Acknowledgments}

This work was conducted in the framework of the DLR project CENT. 


\section{References}

${ }^{1}$ Blake, W. K., Mechanics of flow-induced sound and vibration, Academic Press, Inc., 1986.

${ }^{2}$ Goody, M., "Empirical spectral model of surface pressure fluctuations." AIAA Journal, Vol. 42, No.9, 2004.

${ }^{3}$ Corcos, G. M., "The structure of the turbulent pressure field in Boundary Layer Flows." J. Fluid Mech., Vol. 18, 1964, pp. 353-378.

${ }^{4}$ Willmarth, W. W. and Wooldridge, C. E., "Measurements of the fluctuating pressure at the wall beneath a thick turbulent boundary layer." J. Fluid Mech., Vol. 14, 1962, pp. 187-210.

${ }^{5}$ Bull, M. K., "Wall pressure fluctuations associated with subsonic turbulent boundary layer flow." J. Fluid Mech., Vol. 28, 1967, pp. 719-754.

${ }^{6}$ Blake, W. K., "Turbulent boundary layer wall pressure fluctuations on smooth or rough walls." J. Fluid Mech., Vol. 44(4), 1970, pp. 637-660.

${ }^{7}$ Farabee, T. M. and Casarella, M. J., "Spectral features of wall pressure fluctuations beneath turbulent boundary layers." Phys. Fluids, Vol. A3(10), 1991, pp. 2410-2420.

${ }^{8}$ Arguillat, B., Ricot, D., Robert, G., and Bailly, C., "Measurements of wavenumber-frequency spectrum of wall pressure fluctuations under turbulent flows." AIAA Paper, 2005.

${ }^{9}$ Ehrenfried, K. and Koop, L., "Experimental study of pressure fluctuations beneath a compressible turbulent boundary layer." AIAA Paper, 2008.

${ }^{10}$ Spalart, P. R., "Direct simulation of a turbulent boundary layer up to $\operatorname{Re}_{\theta}=1410 . "$ J. Fluid Mech., Vol. 187, 1988, pp. 61-98.

${ }^{11} \mathrm{Kim}$, J., "On the structure of pressure fluctuations in simulated turbulent channel flow." J. Fluid Mech., Vol. 205, 1989, pp. 421-451.

${ }^{12}$ Choi, H. and Moin, P., "On the space-time characteristics of wall-pressure fluctuations." Phys. Fluids, Vol. A2(8), 1990, pp. 1450-1460.

${ }^{13}$ Viazzo, S., Dejoan, A., and R.Schiestel, "Spectral features of the wall-pressure fluctuations in turbulent wall flows with and without perturbations using LES." J. Heat and Fluid Flow, Vol. 22, 2001, pp. 39-52.

${ }^{14}$ Gloerfelt, X. and Berland, J., "Direct computation of turbulent boundary layer noise." AIAA Paper, 2009.

${ }^{15} \mathrm{Hu}$, N., Reiche, N., and Ewert, R., "Simulation of turbulent boundary layer wall pressure fluctuations via Poisson equation and synthetic turbulence." (submitted to J. Fuild Mech., 2016).

${ }^{16}$ Hockney, R. W. and Eastwood, J. W., Computer simulation using particles, Taylor \& Francis, Inc., 1988.

${ }^{17} \mathrm{Hu}, \mathrm{N}$. and Herr, M., "Characteristics of wall pressure fluctuations for a flat plate turbulent boundary layer with pressure gradients." AIAA Paper, 2016.

${ }^{18}$ Ewert, R., Dierke, J., Siebert, J., Neifeld, A., Appel, C., Siefert, M., and Kornow, O., "CAA broadband noise prediction for aeroacoustic design." J. Sound Vib., Vol. 330, 2011, pp. 4139-4160.

${ }^{19}$ Ewert, R., "Broadband slat noise prediction based on CAA and stochasic sound sources from a fast random particlemesh (RPM) method." Computers \& Fluids, Vol. 37, 2008, pp. 369-387.

${ }^{20}$ Togiti, V. and Eisfeld, B., "Assessment of g-equation formulation for a second-moment Reynolds stress turbulence model." AIAA Paper, 2015.

${ }^{21}$ Bailly, C. and Juvé, D., "A stochastic approach to compute subsonic noise using linearized Euler's equations." $A I A A$ Paper, 1999.

${ }^{22}$ Wooldridge, C. E. and Willmarth, W. W., "Measurements of the correlation between the fluctuating velocities and the fluctuating wall pressure in a thick turbulent boundary layer," Tech. rep., The university of Michigan, Department of Aeronautical and Astronautical Engineering, 1962. 